\title{
Morphological characteristics of monosodium urate: a transmission electron microscopic study of intact natural and synthetic crystals
}

\author{
HERNANDO PAUL, ANTONIO J.REGINATO,AND \\ H. RALPH SCHUMACHER \\ From the University of Pennsylvania School of Medicine and Veterans Administration Medical Center, \\ Philadelphia, PA 19104, USA
}

SUMmaRY Transmission electron microscopic studies of synthetic and natural monosodium urate crystals dried on formvar coated grids showed identical internal structures in all crystals. At higher magnification the crystals' surface showed angular or wavy irregularities, and more rarely some crystals appeared to have other tiny crystals on the surface. Protein-like surface coating was not observed except in crystals from one asymptomatic patient in whom synovial fluid was loaded with monosodium urate crystals, but no inflammatory cells were present. Heated synthetic monosodium urate crystals retained the ultrastructural characteristics in their interior but they lost their needle or rod-like shape. Transmission electron microscopic study of monosodium urate crystals dried on formvar coated grids provides a quick method of investigating crystal ultrastructure.

Monosodium urate (MSU) crystal structure as seen by compensated polarised light microscopy has been described in detail, ${ }^{1}$ but routine transmission electron microscope (TEM) studies of thin sections of the synovial membrane and tophi have failed to show the ultrastructure of these crystals. ${ }^{23}$ Since MSU crystals are dissolved out during tissue processing for TEM, only crystal outlines have been observed. Recent studies of crystal surface-protein interactions, ${ }^{34}$ adsorption of lysosomal enzymes to crystals, ${ }^{5}$ and nucleation of MSU crystals ${ }^{6}$ suggest that further knowledge of the ultrastructure of MSU might be helpful in understanding the pathogenesis of gout. However, only very few TEM studies have been undertaken on intact MSU crystals. In 1967 Riddle et al. briefly described the electron microscope appearance of such crystals and the alteration of the internal structure by electron beam exposure. ${ }^{7}$ More recently Pritzker et al., using scanning and TEM techniques, reported that the internal architecture of urate was different in synovial fluid (SF) and tophi. The ultrastructure of crystals in tophi suggested the presence of nucleating material. ${ }^{8}$

Accepted for publication 22 January 1982.

Correspondence to Professor A. J. Reginato MD. Veterans Administration Medical Center, University and Woodland Avenues, Philadelphia. PA 19104, USA.
In this report we describe the ultrastructure of heated and unheated intact synthetic MSU crystals and our findings on natural MSU from SF, bursae, and tophi. The external appearance of synthetic crystals was altered by heat. A similar internal architecture was found in synthetic crystals and natural crystals from SF, bursae, and tophi. Most MSU crystals had smooth surfaces with evidence of thick superficial coating on intact crystals from only one synovial fluid.

\section{Materials and methods}

MSU crystals were obtained from 15 synovial fluids, 3 olecranon bursal fluids, one tophus of the helix of the ear, and one tophus adjacent to a knee joint. Ten synovial fluid specimens were aspirated from acutely inflamed and 4 from chronically inflamed joints. One SF was from an asymptomatic joint of a patient with a massive amount of MSU crystals in the synovial membrane and synovial fluid but without an acute inflammatory reaction.

In order to compare the ultrastructure of natural MSU crystals with synthetic crystals, samples of synthetic MSU were prepared in water by the methods of Denko and Whitehouse ${ }^{9}$ and of McCarty and Faires. ${ }^{10}$ Crystals were examined after being heated at $180^{\circ} \mathrm{C}$ for 3 hours and unheated. 


\section{Paul, Reginato, Schumacher}

One drop aspirated from the samples containing MSU crystals was promptly placed on a clean glass slide. Six formvar-coated EM grids were floated on the drop for 10 seconds. To obtain samples with thickness suitable for TEM studies excessive material on the grids was removed gently with the use of filter paper. The grids were then air dried and observed with compensated polarised light microscopy. Those containing negatively birefringent crystals were examined unstained in a Zeiss EM 10 TEM with a 60 $\mathrm{kV}$ beam. The morphology of MSU crystals was studied in over 200 photomicrographs.

Four samples of natural MSU crystals and one of heated synthetic crystals were studied by $x$-ray diffraction. A Debbye-Scherrer powder camera (North American Phillips) of $114.6 \mathrm{~mm}$ diameter with chromium $\mathrm{K}$ alpha radiation and a vanadium filter was used. Measurements were based on wave length $2 \cdot 29092 \AA(22.9092 \mathrm{~nm})$. The specimens were exposed for 20 hours at $60 \mathrm{kV}$ and $20 \mathrm{~mA}$. All 5 specimens studied showed exclusively MSU crystals.

\section{Results}

NATURAL MSU CRYSTALS

Urate crystals including a range varying in size, shape, and ultrastructure were seen in each individual sample. Birefringence by polarised light was strong or weakly negative. Similar general findings were observed in SF, bursae, and tophi (Fig. 1). Although variation in size had been appreciated by polarised light microscopy, the range of different shapes and extremes of small size had not been readily detectable.
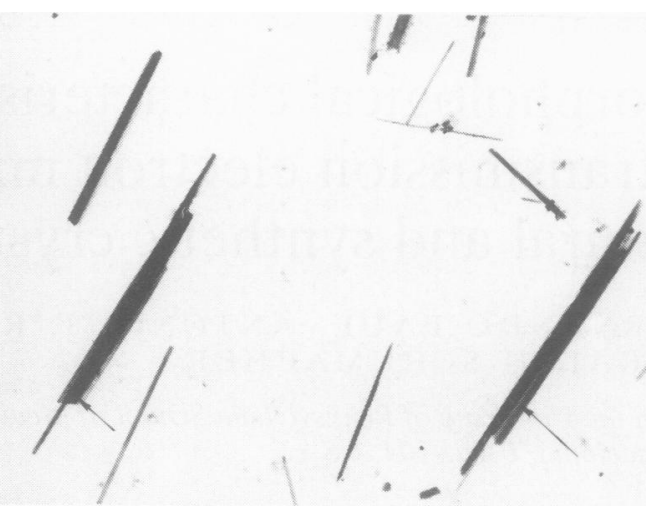

Fig. 1 Ear tophus. MSU crystals exhibiting variable shape, size, and electron density. These are similar to those $M S U$ observed in synovial fluid and articular tophi. Stacking of crystals is seen at the arrows. $(\times 2860)$.

Sizes varied considerably, but there was a tendency for the crystals to be smaller in SF. Crystal length in general ranged from 1 to $40 \mu \mathrm{m}$ in bursae and tophi, while it was between 1 and $20 \mu \mathrm{m}$ in SFs. Mosto crystals were long with parallel sides and 2 blunt ends (Fig. 2A). Other crystals were long with parallel sideso and one somewhat less blunt end, though ends were? not truly needle-like (Fig. 2B). A few crystals were very short $(0.5 \mu \mathrm{m})$ and rod-shaped with 2 blunt ends. A common finding was stacking together of 2 or more crystals (Figs. 1,2B). Some of these twinned or stacked crystals appeared to have structural continuity between individual crystals. In other stacked

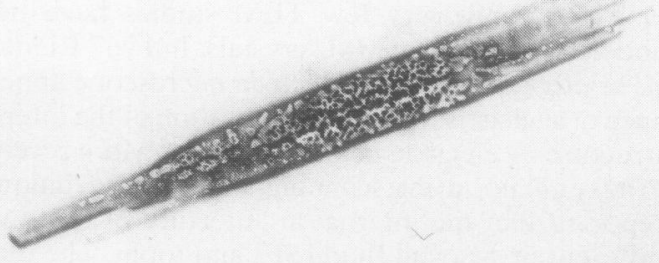

Fig. 2 Different shapes and sizes of MSU crystals. 2A. Most MSU crystals were long with parallel sides and blunt ends. Olecranon bursal fluid $(\times 12$ 900). 2B. Other MSU crystals exhibiting parallel sides with one blunt end and others less blunt but not truly needle-like. Apparent stacking of crystal components is also seen, with continuity of crystal structure in the centre. Synovial fluid $(\times 10300)$. 
forms this appearance seemed to arise from superimposition of crystals.

The crystal surface was most often smooth at lower magnifications (Figs. 1, 2), but at higher magnifications angular or curved irregularities of the crystal surface were observed in most SF, bursae, and tophi (Figs. 3A, 3B). Some crystals appeared to have other tiny crystals on the surface (Fig. 3C). A few of those tiny crystals exhibited apparent continuity with the larger crystal. Others had different ultrastructure and were actually morphologically reminiscent of calcium pyrophosphate dihydrate (CPPD) because of their greater density with round and more uniformly arranged holes. ${ }^{11}$ The latter ultrastructurally different crystals were observed in bursae (Fig. 3C) and SF but not in the 2 tophi examined.

Diligent examination of the surface failed to reveal any evidence of definite protein-like coating on most crystals. A thick fluffy coating was observed, however, on MSU crystals in the SF from the one patient who was asymptomatic (and without SF inflammatory cells) (Fig. 3D). Many crystals had a sharply demarcated, less dense homogeneous surface layer that might possibly be a coating or more likely is merely one appearance of the surface crystal material. There was partial disappearance of the fluffy coating after treatment with hyaluronidase, but not of the homogeneous material on other crystals.

The internal architecture as seen by TEM also varied considerably. MSU crystals showing different grades of electron density and variable internal architecture were observed in all specimens of SF, bursae, or tophi (Figs. 2A, 4C). The interior of the crystals developed an electron-lucent network which was artefactually induced by exposure to the electron beam. At the very beginning of electron beam exposure the crystals appeared uniformly electrondense (Fig. 4A). Within a few seconds many small electron-lucent areas were observed (Fig. 4B). Some crystals appeared to become stabilised and showed no further changes, even after prolonged beam exposure (Figs. 2A, 3C, 3D). This was usually true of the smaller and/or thinner crystals. In larger and thicker crystals the electron lucencies enlarged while
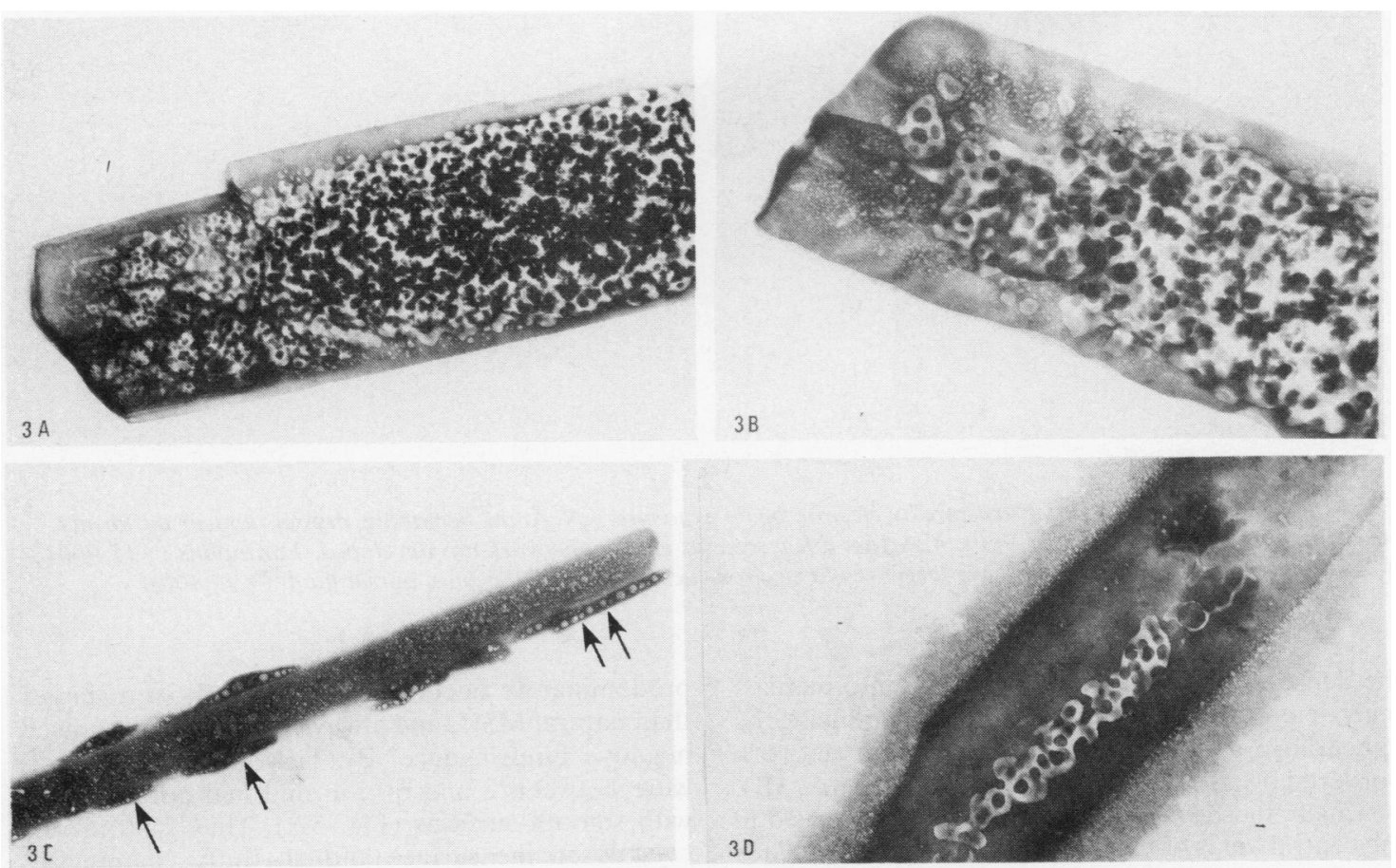

Fig. 3 Surface characteristic and coating of MSU crystals. 3A. MSU crystal showing less dense homogeneous surface with angular irregularities of the surface. Synovial fluid. $(\times 17950)$. 3B. MSU crystal with wavy irregularities on the surface. Synovial fluid. $(\times 2780)$. 3C. MSU crystal with tiny crystals on its surface. Some satellite crystals exhibit internal structure like MSU crystals and showed structural continuity with the large crystals (arrow), while others appear to be only attached to the surface (double arrow). Olecranol bursal fluid $(\times 17950)$. 3D. Abundant granular coating on MSU crystal in the synovial fluid of an asymptomatic gouty patient with massive amounts of urate crystals in the synovial fluid without inflammatory cells $(\times 36800)$ 


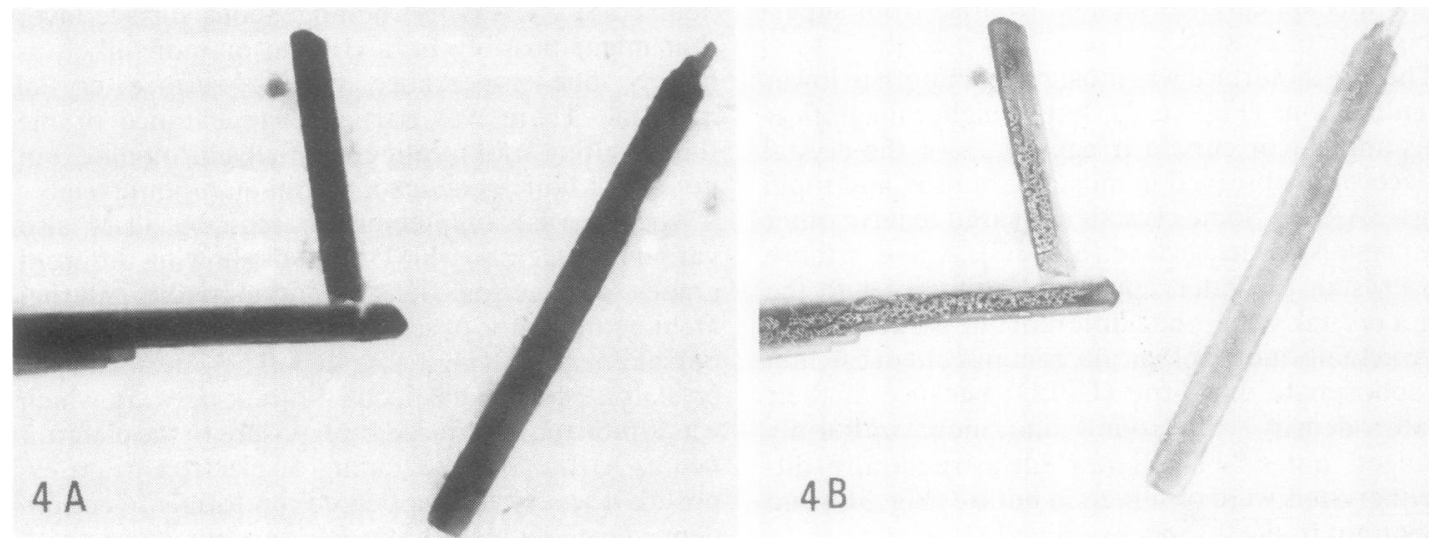

4

Fig. 4 Changes on MSU crystal structure by electron beam exposure. 4A. At the beginning, crystals appear uniformly electron-dense. Ear tophus. $(\times 15$ 900). 4B. After a few seconds a lucent network has developed. Ear tophus $(\times 15900)$. 4C. Rarely apparent MSU crystals have larger ovoid electron-lucent spaces. Olecranon bursal fluid. $(\times 99400)$.

the dense areas appeared to aggregate into increasingly dense round or oblong structures, producing the appearance of an 'electron lucent tubular network bordered by an electron dense shell' ${ }^{\text {' }}$ (Figs. 3A, 3B). Normally these structural changes were observed in the central areas of the crystal, while the crystal ends developed only smaller electron lucencies. In bursae very occasional crystals showed larger ovoid electron-lucent spaces (Fig. 4C).

SYNTHETIC MSU CRYSTALS

Under polarised light crystals synthesised over 72 hours by the methods previously mentioned were predominantly needle or rod shaped, were larger than natural MSU, and always very strongly showed negative birefringence. By TEM synthetic crystals were between 2 and $60 \mu \mathrm{m}$ and had parallel sides with smooth surfaces (Fig. 5A). They had no tiny crystals on the surface and no fluffy coating was observed. They showed variable density and developed structural changes induced by the electron beam exposure similar to those of natural MSU crystals. Heated MSU crystals retained their ultrastructural characteristics in their interior, but they lost their needle- or rod-like shapes, and the surface became very irregular (Fig. 5B). A homogeneous 

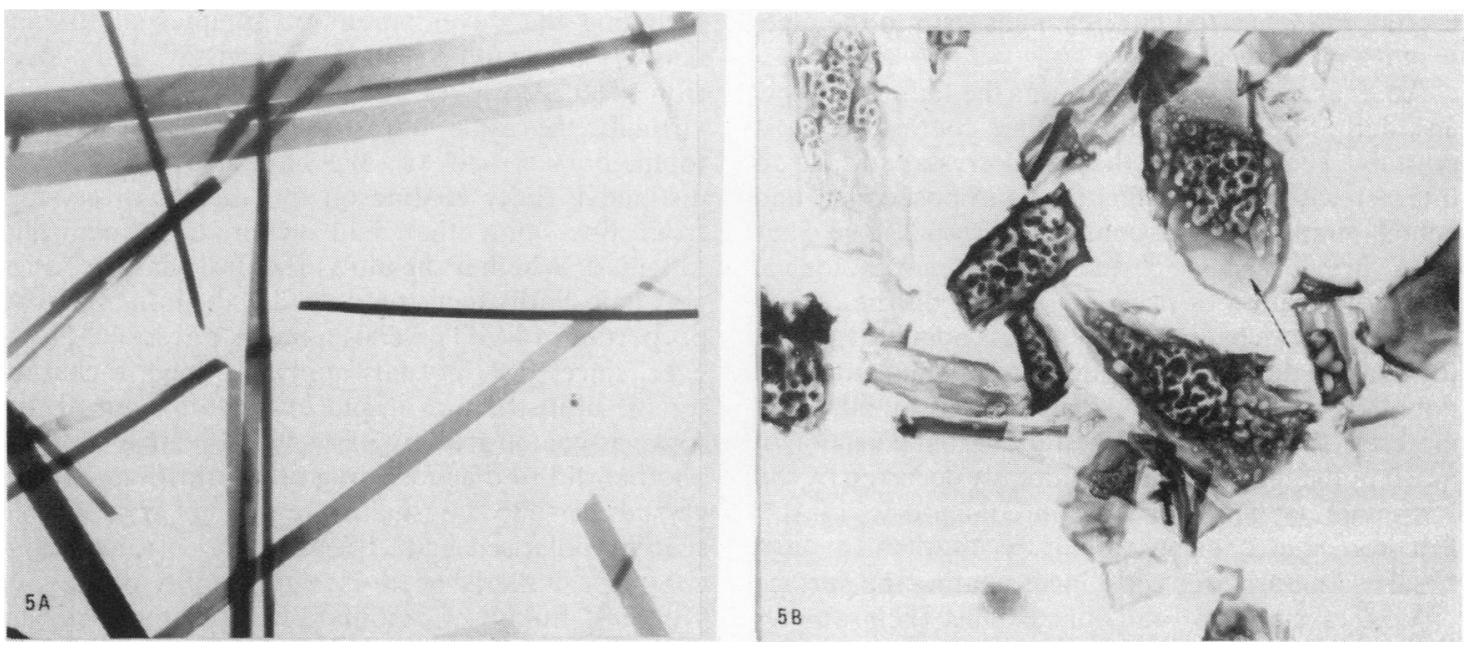

Fig. 5 Synthetic MSU crystals. 5A. Synthetic MSU crystals are larger than natural crystals. At low magnification they exhibit smooth surfaces and needle-like or rod shapes. Electron micrograph taken after minimal exposure to electron beam. $(\times 3370)$. 5B. Heated synthetic MSU crystals retain the internal ultrastructural characteristics, but they acquire a bizarre appearance and irregular surface and lose their well defined rod-or needle-like shapes. The homogeneous surface laye'r is seen at the arron:

lighter surface structure, such as was seen on some natural crystals, occurred on some of the synthetic crystals with or without heating (Fig. 5B).

\section{Discussion}

The present study provides further information on the morphological characteristics of natural and synthetic MSU crystals. These crystals and other water soluble crystals such as steroid crystals ${ }^{12}$ and cholesterol crystals $^{13}$ are usually lost during tissue processing, and only an electron-dense outline of the crystal remains. CPPD and calcium oxalate ${ }^{14}$ are not dissolved, but sections for TEM might cut through crystals making shape an imprecise criterion for their identification. By using the formvar-coated grid technique, crystal dissolution is avoided and crystals retain their actual shapes. CPPD crystals continue being electron-dense and diffusely foamy with round, more uniformly distributed holes, ${ }^{15}$ and are thus similar in fine structure to calcium oxalate. ${ }^{14}$ Cholesterol and steroid crystals appear uniformly electron-dense with almost no changes induced by the beam exposure. Thus all these crystals have their own characteristics, which are different from those of MSU crystals. The occasional atypical crystals seen in bursae (Figs. 3C, 5C) are still unexplained and might represent different morphology of MSU or minute amount of some other crystal missed by $x$-ray diffraction analysis.

MSU crystals in our study showed some variability in shape and internal architecture. The same range of findings was consistently observed in SF, bursae, tophi, and synthetic crystals, indicating that MSU crystals formed or found under different conditions had a distinct ultrastructural resemblance. Previous observations had suggested that urates precipitated in tophi and SF appeared different. ${ }^{*}$ We were able to observe similar crystals to those described by these authors, but they were seen in specimens from SF, bursae, or tophi. Since only few tophi were studied by Pritzker et al. ${ }^{8}$ and by us, the differences might represent sampling of crystals predominantly at different stages of crystal growth or variations in individual patients, as well as different techniques of processing crystals. In the present study the MSU crystal size in SF was slightly smaller than in tophi or bursae. Smaller crystals have also been observed in MSU synthesised in SF than in water. ${ }^{16}$ Factors such as lysis of urate crystals by neutrophil peroxidase ${ }^{17}$ or other factors increasing MSU solubility ${ }^{18}$ may also be responsible for the smaller size of MSU crystals in SF.

The different grades of electron density and internal architecture observed in our study appeared to result from the variability of crystal size and shape. Thinner crystals seemed to respond differently to the electron beam. The variable ultrastructural network shown here was clearly an artefact induced by the electron beam exposure. We have, however, demonstrated in other studies that nonartefactual holes are identifiable during the early growth of MSU crystals. ${ }^{16}$ Whether these holes during growth, and any nucleating material that may be trapped in the 
crystal, influence the later changes seen in the electron beam is not known.

An interesting observation was the failure to show any definite or significant surface coating on most crystals. The ability of the MSU crystal surface to interact with proteins has been proposed, ${ }^{19-21}$ and actual immunoglobulin coating on crystals in vivo has been previously suggested. ${ }^{34}$ But morphological proof of location of immunoglobulin on the crystal surface had not been presented. It is possible that the formvar-coated grid techniques used in our study did not allow visualisation of small amounts of adherent material on the surface of crystals, or that digestion of much of the protein coat had already occurred by the lysosomal enzymes in the inflammatory fluids. ${ }^{22}$ Pritzker et al., ${ }^{,}$using similar techniques to ours, observed amorphous substances coating the surface of MSU crystals in SF and bursae. Since their samples were preserved in ethanol, these authors interpreted their findings as precipitated 'mucin'. It is intriguing that they observed smooth surfaced crystals on the joint surfaces of asymptomatic gouty patients, while in our study, on the contrary, the only SF in which a definite crystal surface coat was observed came from an asymptomatic gouty patient. What this fluffy coating contains and whether it is what prevented inflammation remains to be determined. Whether the less dense homogeneous material on the surface of many crystals is only part of the crystal wall or may include coating material will be studied in the future with immuno EM techniques.

Some very small crystals were also observed in fluids from our patients. Many were on the surface of other crystals; we do not know the exact nature of these crystals. $X$-ray diffration in one fluid containing such crystals showed only MSU, but very small crystals may not allow electron diffraction or give off sufficient $x$-rays for elemental analysis. Thus it is possible that they represent urate or other unidentified crystals, such as even CPPD or other calcium salts.

The study of synthetic MSU crystals clearly showed that heating may induce morphological changes, especially on the crystal surface, destroying the well-defined needle-like or rod shapes. Nevertheless, the internal architecture of heated crystals appeared similar to unheated crystals. Mandel, using $x$-ray diffraction and thermal gravimetric analysis, has postulated that heating of MSU crystals induces changes in the chemical composition and crystal structure. ${ }^{23}$

The present studies demonstrate that drying very small amounts of fluids on formvar-coated grids allows visualisation of whole MSU crystal ultrastructure. Some of our observations confirm the findings previously reported by Pritzker and his co-workers, including the development of an internal tubular network that can be seen after exposure to the electron beam. However, in contrast to them we found virtually the same ultrastructure of crystals from tophi, bursae, and SF. We have shown, so far, a distinctive fluffy coating on the surface of crystals from one joint that had never been clinically inflamed. Whether the ultrastructural characteristics may help clarify factors involved in the inflammatory properties of MSU crystals remains uncertain. However, since MSU crystals appear to have a characteristic ultrastructure, rapid examination on SF on formvar-coated grids by TEM, when available, offers another aid to diagnosis in gouty arthritis, when no crystals, or only unusual or very small crystals, are seen by polarised light. ${ }^{24}$ Scanning electron microscopy has similarly been used to identify crystals in synovial fluid. ${ }^{25}$ Combinations of these newer approaches offer the opportunity of many new advances in the understanding of the role of crystals or other particulate matter in arthritis. ${ }^{26-28}$

The authors acknowledge the superb technical assistance of Gilda Clayburne, Susan Rothfuss, Marie Sieck, and the excellent secretarial help of Joanne Loguidice.

The work was supported in part by grants from the McCabe Foundation, Barsumian Fund, Veterans Administration, and from Centro Nacional de Enfermedades Reumaticas MSAS Universidad Central de Venezuela and Fundacion Gran Mariscal de Ayacucho, Venezuela.

\section{References}

1 Phelps P, Steele A D, McCarty D J. Compensated polarized light microscopy. Identification of crystals in synovial fluids from gout and psuedogout. JAMA 1968; 203: 508-12.

2 Agudelo C A, Schumacher H R. The synovitis of acute gouty arthritis. A light and electron microscopic study. Hum Pathol 1973; 4: 265-79.

3 Hasselbacher P, Schumacher H R. Immunoglobulin in tophi and on the surface of monosodium urate crystals. Arthritis Rheum 1978; 21: 353-61.

4 Kozin F, McCarty D J. Protein adsorption to monosodium urate, calcium pyrophosphate dihydrate and silica crystals. Arthritis Rheum 1976; 19: 433-8.

5 Ginsberg M H, Kozin F, Chow D, May J, Skosey J L. Adsorption of polymorphonuclear leukocyte lysosomal enzymes to monosodium urate crystals. Arthritis Rheum 1977; 20: 1538-42.

6 Tak H, Cooper S M, Wilcox W R. Studies on the nucleation of monosodium urate at $37^{\circ} \mathrm{C}$. Arthritis Rheum $1980 ; 23$ : 574-80.

7 Riddle J M, Bluhm G B, Barnhart M I. Ultrastructural study of leukocytes and urates in gouty arthritis. Ann Rheum Dis 1967; 26: $389-401$.

8 Pritzker K P H, Zahn C E, Nyburg S C, Luk S C, Houpt J B. The ultrastructure of urate crystals in gout. $J$ Rheumatol 1978; 5: 7-18.

9 Denko C W, Whitehouse M W. Experimental inflammation induced by naturally occurring microcrystalline calcium salts. $J$ Rheumatol 1976; 3: 54-62.

10 McCarty D J, Faires J S. A comparison of the duration of local anti-inflammatory effect of several adrenocorticosteroid esters. A bioassay technique. Curr Ther Res 1963; 5: 284-90.

11 Schumacher H R. Ultrastructural findings in chondrocalcinosis and pseudogout. Arthritis Rheum 1976; 19: 413-25. 
12 Gordon G V, Schumacher H R. Electron microscopic study of depot corticosteroid crystals with clinical studies after intraarticular injection. $J$ Rheumatol 1979; 6: 7-14.

13 Parker F, Odland G F. Ultrastructural and lipid biochemical comparisons of human eruptive tuberous and planar xanthomas. Isr J Med Sci 1973; 9: 395-423.

14 Hoffman G S, Schumacher H R, Paul H, Cherian V, Ramsey A, Frank W A. Calcium oxalate crystals associated arthritis in chronic renal failure. Arthritis Rheum 1981; 24: S73 (abst).

15 Pritzker N P H, Phillips H, Luk S C, Koven I H, Kiss A, Houpt J B. Pseudotumor or temporomandibular joint destructive calcium pyrophosphate dihydrate arthropathy.J Rheumatol 1976; 3: 70-81.

16 Paul H, Schumacher H R. Sequential ultrastructural changes in the growth of monosodium urate crystals. Arthritis Rheum 1981; 24: 573 (abst).

17 Howell R R, Seegmiller J E. Uricolysis by human leukocytes. Nature 1962; 196: 482-3.

18 Kippen I, Klinenberg J R, Weinberger A, Wilcox W R. Factors affecting urate solubility in vitro. Ann Rheum Dis 1974; 33: 313-7.

19 Kellermeyer R W, Breckenridge R T. The inflammatory process in acute gouty arthritis. Activation of Hageman factor by sodium urate crystals. J Lab Clin Med 1965; 65: 307-15.

20 Naff G B, Byers P H. Complement as a mediator of inflammation in acute gouty arthritis. I. Studies on the reaction between human serum complement and sodium urate crystals.J Lab Clin Med 1973; 81: 747-60.

21 Campion D S, Bluestone R, Klinenberg J R. Displacement by uricosuric agents of sodium urate bound to human serum albumin. Biochem Pharmacol 1974; 23: 1653-7.

22 Wallingford W R, McCarty D J. Differential membranolytic effects of microcrystalline sodium urate and calcium pyrophsophate dihydrate. J Exp Med 1971; 133: 100-12.

23 Mandel N S. Structural changes in sodium urate crystals on heating. Arthritis Rheum 1980; 23: 772-6.

24 Honig S, Gorevic P, Hoffstein S, Weissmann G. Crystal deposition disease. Am J Med 1977; 63: 161-4.

25 Faure G, Netter P, Malman B, Steinmetz J, Duheille J, Gaucher A. Scanning electron microscopic study of microcrystals implicated in human rheumatic diseases. Scanning Electron Microscopy 1980; 3: 163-78.

26 Crocker $P$ R, Dieppe $P$ A, Tyler T, Chapman S K, Willoughby D A. The identification of particulate matter in biological tissues and fluids. J Pathol 1976; 121: 37-40.

27 Crocker P R, Doyle D V, Levison D A. A practical method for the identification of particulate and crystalline material in paraffin-embedded tissue specimens. J Pathol 1980; 131: $165-73$.

28 Ali S Y, Griffiths S. New type of calcium phosphate crystals in arthritis cartilage. Semin Arthritis Rheum 1981; 11: suppl 1: 124-6. 